\title{
INCLUSION OF BIOLOGICAL FACTORS IN PARALLEL-ARCHITECTURE NORMAL-TISSUE COMPLICATION PROBABILITY MODEL FOR RADIATION-INDUCED LIVER DISEASE
}

\author{
Jason Chia-Hsien Cheng, M.D., M.S., ${ }^{* \$}$ Hua-Shan Liu, M.S., ${ }^{*}$ Jian-Kuen Wu, M.S., ${ }^{\ddagger}$ \\ Hsiao-Wen Chung, Ph.D., * and Gwo-Jen Jan, Ph.D.* \\ *Department of Electrical Engineering, National Taiwan University, Taipei, Taiwan; Departments of ${ }^{\dagger}$ Radiation Oncology and \\ ${ }^{\ddagger}$ Medical Physics, Koo Foundation Sun Yat-Sen Cancer Center, Taipei, Taiwan; and ${ }^{\S}$ Cancer Research Center and \\ Division of Radiation Oncology, Department of Oncology, National Taiwan University Hospital and \\ National Taiwan University College of Medicine, Taipei, Taiwan
}

\begin{abstract}
Purpose: To include biologic factors in parallel-architecture normal-tissue complication probability (NTCP)

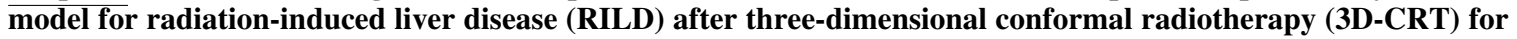
gastric or hepatic cancer.

Methods and Materials: A total of 151 patients (89 with hepatocellular carcinoma and 62 with gastric cancer) who received 3D-CRT to the liver were included (isocenter dose range 33.0 to 66.0 Gy; mean 48.0 Gy). RILD was defined as grade 3 or higher liver toxicity according to Common Toxicity Criteria Version 2.0 of the National Cancer Institute within 4 months after 3D-CRT. Possible correlations of patient-related or dosimetric factors with RILD were tested. Maximum-likelihood analysis estimated NTCP model parameters for group and subgroups. Goodness-of-fit analysis estimated deviance of NTCP model parameters between subgroups.

Results: RILD developed in 25 patients. Hepatitis B virus carrier status $(p<0.001)$ was the only significant independent factor. The 4 parallel NTCP model parameters, mean functional reserve $\left(V_{50}\right)$, width of functional reserve distribution $(\sigma)$, dose damage to $50 \%$ of liver subunits $\left(D_{50}\right)$, and slope parameter for subunit doseresponse $(k)$, were respectively, $0.54,0.14,50 \mathrm{~Gy}, 0.18$ (group); $0.53,0.07,50 \mathrm{~Gy}, 4.6 \times 10^{-7}$ (carriers); 0.59, 0.12, 25 Gy, 59.8 (noncarriers). In carrier-state subgroups, goodness-of-fit deviance with 1 subgroup's parameter set would have been worse in the other group. Across subgroups, patients with RILD all had liver fraction damage (f) greater than 0.4 compared with wider distribution for the whole group.

Conclusions: RILD is described with a parallel-architecture NTCP model for HBV carriers and noncarriers with a threshold effect greater than 0.4. The main difference is in slope parameter for subunit dose-response. (C) 2005 Elsevier Inc.
\end{abstract}

Radiation-induced liver disease, Normal-tissue complication probability, Hepatitis B virus, Parameterization.

\section{INTRODUCTION}

Radiation-induced liver disease (RILD) has been one of the most serious complications in patients with hepatic malignancies undergoing three-dimensional conformal radiotherapy (3D-CRT) $(1,2)$. Both clinical and dosimetric indicators are used to predict development of RILD $(3,4)$. Normal-tissue complication probability (NTCP) models, including the Lyman NTCP model and the parallel-architecture NTCP model, have been tested to calculate the risk of radiation-related complications (5-8). The parallel-architecture NTCP model plays certain roles in describing radiation-induced parallel-type organ damage $(7,8)$. Nondosimetric biologic factors have been found to have an impact

Reprint requests to: Gwo-Jen Jan, Ph.D., Department of Electrical Engineering, National Taiwan University, No. 1, Sec. 4, Roosevelt Road, Taipei 106, Taiwan. Tel: (+886) 2-2363-5251; Fax: (+886) 2-2363-8247; E-mail: jasoncheng@ ha.mc.ntu.edu.tw This work was supported by the National Science Council, on NTCP parameterization (5). Our previous data showed that application of the Lyman NTCP method is feasible in patients with RILD when biologic factors are included into the modeling process (6). The current study was designed to investigate inclusion of biologic factors as part of the parallel-architecture NTCP model for patients with hepatocellular carcinoma (HCC) or gastric carcinoma (GC) undergoing 3D-CRT to predict the risk of RILD.

\section{METHODS AND MATERIALS}

\section{Patients and treatments}

From 1993 to 2001, a total of 175 patients underwent hepatic irradiation at our institution. Among them, 104 patients were

Executive Yuan, Taiwan, ROC (grant number: NSC 93-2314-B002-194) and the research grant NTUH 93N02, National Taiwan University Hospital.

Received Sep 15, 2004 and in revised form Nov 16, 2004. Accepted for publication Dec 3, 2004. 
diagnosed with HCC and 71 patients were diagnosed with GC. Of the $104 \mathrm{HCC}$ patients, 15 were excluded for incomplete dosevolume data for their palliative treatment (10 patients) or insufficient follow-up of less than 4 months after completion of 3D-CRT (5 patients). Of $71 \mathrm{GC}$ patients, 9 were excluded for major deviation in chemotherapy and laboratory data from the radiation treatment protocol (described below). Thus, a total of 151 patients formed the basis of this study, including 89 patients with HCC and 62 patients with GC. Of the total, 104 patients were male and 47 were female. Child-Pugh Class A cirrhosis of the liver existed in 130 patients, and 21 patients had Class B cirrhosis. Chronic HBV carriers (defined as positive serum hepatitis B surface antigen greater than $4 \mathrm{ng} / \mathrm{mL}$ by microparticle enzyme immunoassay) accounted for 76 of the 151 patients, including $65 \mathrm{HCC}$ patients and 11 GC patients. Patient characteristics are shown in Table 1.

No patient was given radiation to the whole liver. Radiation treatment technique and dose-volume criteria in patients with HCC have been reported previously (9). Briefly, gross tumor volume was defined as the hepatic tumor volume visualized by 3D computation of contrast CT-defined contours. Respiratory movement estimated fluoroscopically in simulation was taken into account for planning of target volume. Nonneoplastic normal liver tissue was defined as total-liver volume minus gross tumor volume. Radiation portals were generally designed to include the gross tumor volume with $1.5-\mathrm{cm}$ to $2-\mathrm{cm}$ margins, with specific modifications to cover the planning target volume. Radiation therapy was delivered by a 6-MV or 18-MV linear accelerator. Mean dose of radiation to the isocenter was $49.9 \pm 6.2 \mathrm{~Gy}$ (range, $36.0-66.0 \mathrm{~Gy}$ ). All but 3 patients had a daily fraction of 1.8 to 2 Gy. Two of these patients received a daily fraction size of $3 \mathrm{~Gy}$, and the other patient received $2.5 \mathrm{~Gy}$, all 3 of which converted to the normalized isobiologic effective dose of 2 Gy per fraction according to a linear quadratic model with $\alpha / \beta$ ratio of 2 Gy for dosimetric computation $(5,7)$. The spatial design of the coplanar and noncoplanar beam combination was made by use of the beam's eye view reconstructed from a CT-based simulation. In addition to 3D-CRT, $74 \mathrm{HCC}$ patients underwent transcatheter arterial chemoembolization (TACE). This group included 39 patients who received TACE before $3 \mathrm{D}-\mathrm{CRT}, 7$ who received TACE after 3D-CRT, and 28 who received TACE both before and after 3D-CRT. An interval of at least 30 days between TACE and 3D-CRT was necessary regardless of which treatment was given first to allow adequate recovery of hepatic function.

Radiation treatment in patients with GC usually started 6 to 8 weeks after gastrectomy. The radiotherapy protocol of postgastrectomy adjuvant treatment was similar to that in the Intergroup 0116 Gastric Surgical Adjuvant Trial (10), including a median dose of 45 Gy (range, 41.4-57.1 Gy) in daily fractions of 1.8-2 Gy, with a 6-MV (mainly in the anterior-posterior field) or a 18-MV photon beam directed to the tumor bed, regional lymph nodes, and 1.5 to 2 $\mathrm{cm}$ beyond the proximal and distal margins of resection. Two-field (anterior-posterior/posterior-anterior), three-field (anterior-posterior/ bilateral opposed with wedge pairs), or four-field box beam designs were used for the treatment. Radiation portals were designed by a CT-based treatment-planning system (FOCUS system, CMS Co., St. Louis, MO) that generated complete dose-volume histograms (DVHs) of targets and organs at risk. The chemotherapy protocol for GC patients included a weekly cycle of high-dose 5-fluorouracil (median, $2000 \mathrm{mg} / \mathrm{m}^{2}$; range, 1,400-2,600 mg/m $\mathrm{m}^{2}$ ) and leucovorin $\left(300 \mathrm{mg} / \mathrm{m}^{2}\right)$ given as a 24-hour continuous intravenous infusion concurrently with radiotherapy. Concomitant chemotherapy was given for a median of 5 cycles (range, 1-6).

\section{RILD definition}

Patients were monitored weekly during the 3D-CRT, with historytaking, physical examination, blood counts, and blood chemistries that included liver function tests. Each patient was followed monthly after completion of 3D-CRT for at least 4 months. RILD was defined as Grade 3 or higher hepatic toxicity as described by the Common Toxicity Criteria Version 2.0 of the National Cancer Institute (11) that developed within 4 months after completion of 3D-CRT. Progressive disease was assessed by imaging studies and by paracentesis of ascites if sufficient fluid were present. Some patients had elevated serum concentrations of transaminases compatible with the activity level of their malignant disease before 3D-CRT began. The hepatic toxicity of these patients was determined on the basis of transaminase levels elevated to at least 5 times the pretreatment baseline rather than the upper limits of the normal range.

\section{Parallel-architecture NTCP parameterization}

The four-parameter parallel-architecture NTCP model, first established by Jackson and colleagues (12), was used in this study to describe the probability of RILD. In the model,

$$
H(f)=N T C P=\frac{1}{\sqrt{2 \pi \sigma_{v}^{2}}} \int_{o}^{f} \exp \left[-\left(v-v_{50}\right)^{2} / 2 \sigma_{v}^{2}\right] d v
$$

with $\mathrm{v}$ the partial volume that is irradiated. $\mathrm{V}_{50}$ is the mean value of the functional reserve, and $\sigma_{v}$ is the width of the functional reserve distribution.

The probability of damaging a subunit at a given biologically equivalent dose is described as

$$
\mathrm{P}(\mathrm{d})=1 /\left[1+\left(\mathrm{d}_{1 / 2} / \mathrm{d}\right)^{\mathrm{k}}\right]
$$

Two parameters, $\mathrm{D}_{50}$ and $k$, are defined as the dose that causes damage to half of the functional subunits and the slope parameter of the subunit dose-response curve, respectively.

Data from the differential dose-volume histogram yields summed fractions of organ damaged at each dose level as

$$
\mathrm{f}=\sum \mathrm{v}_{\mathrm{i}} \mathrm{P}\left(\mathrm{d}_{\mathrm{i}}\right)
$$

where $\mathrm{v}_{i}$ and $\mathrm{D}_{i}$ are the fraction volume and the corresponding dose derived from the differential dose-volume histogram. Therefore, the 4-parameter $\left(\mathrm{v}_{50}, \sigma_{\mathrm{v}}, \mathrm{D}_{50}, k\right)$ parallel-architecture NTCP is expressed as

$$
\mathrm{NTCP}=\mathrm{H}\left(\sum \mathrm{v}_{\mathrm{i}} \mathrm{P}\left(\mathrm{d}_{\mathrm{i}}\right)\right)
$$

The maximum-likelihood method is used to fit the model to the complication data. The best model parameters are obtained by maximization of the model probability that the observed pattern of RILD occurs. The likelihood function is defined as the calculated NTCPs for patients with RILD $\times(1-\mathrm{NTCP})$ for all patients without RILD. Confidence intervals for the parameters are derived by the profile-likelihood method $(13,14)$.

\section{Statistical analysis}

Patient-related, treatment-related, and dosimetry-related factors were first analyzed for correlation with RILD. In univariate analysis, the Student's $t$ test was used to compare continuous variables 
Table 1. Patient characteristics $(n=151)$

\begin{tabular}{|c|c|c|}
\hline Categorical variable & $\mathrm{HCC}$ & $\mathrm{GC}$ \\
\hline \multicolumn{3}{|l|}{ Gender } \\
\hline Male & 64 & 40 \\
\hline Female & 25 & 22 \\
\hline \multicolumn{3}{|l|}{ AJCC Stage } \\
\hline IB & 0 & 4 \\
\hline II & 27 & 14 \\
\hline III & 11 & 29 \\
\hline IV & 51 & 15 \\
\hline \multicolumn{3}{|l|}{ T stage } \\
\hline $\mathrm{T} 1$ & 0 & 3 \\
\hline $\mathrm{T} 2$ & 26 & 27 \\
\hline T3 & 13 & 60 \\
\hline $\mathrm{T} 4$ & 50 & 10 \\
\hline \multicolumn{3}{|l|}{$\mathrm{N}$ stage } \\
\hline No & 86 & 13 \\
\hline N1 & 3 & 45 \\
\hline $\mathrm{N} 2$ & 0 & 26 \\
\hline N3 & 0 & 16 \\
\hline \multicolumn{3}{|l|}{ M stage } \\
\hline M0 & 81 & 62 \\
\hline M1 & 8 & 0 \\
\hline \multicolumn{3}{|l|}{$\begin{array}{l}\text { Chronic hepatitis B viral } \\
\text { carrier }\end{array}$} \\
\hline Yes & 65 & 11 \\
\hline No & 24 & 51 \\
\hline \multicolumn{3}{|l|}{$\begin{array}{l}\text { Child-Pugh classification for } \\
\text { cirrhosis of liver }\end{array}$} \\
\hline A & 68 & 62 \\
\hline B & 21 & 0 \\
\hline Continuous variable & Mean $\pm \mathrm{SD}$ (range) & Mean \pm SD (range) \\
\hline Age & $56.5 \pm 13.1(19.4-82.2)$ & $52.9 \pm 12.5(21.5-80.9)$ \\
\hline Isocenter dose (Gy) & $49.9 \pm 6.2(36.0-66.0)$ & $45.4 \pm 1.9(41.4-57.1)$ \\
\hline Gross tumor volume $(\mathrm{mL})$ & $683.7 \pm 737.9(7-4529)$ & NA \\
\hline Normal liver volume (mL) & $1153.4 \pm 350.2(614-2907)$ & $1157.6 \pm 242.0(707-1791)$ \\
\hline
\end{tabular}

Abbreviations: AJCC $=$ American Joint Committee on Cancer; GC = gastric carcinoma; $\mathrm{HCC}=$ hepatocellular carcinoma; NA = not applicable due to adjuvant setting of radiotherapy; SD = standard deviation.

between patients with and without RILD, whereas the chi-square test or Fisher's exact test was used to compare categorical variables. Logistic regression analysis, if more than 1 factor reached significance in the univariate test, was used to investigate multivariate impact on the occurrence of RILD. A $p$ value of less than 0.05 resulting from two-sided tests was considered statistically significant. The adjusted NTCP parameters were again obtained by subgrouping patients on the basis of a nondosimetric factor that was significantly correlated with RILD. Goodness-of-fit analysis was used to estimate both the deviances of NTCP model parameters of the subgroups from the parameters of the entire group and deviances of model parameters between subgroups.

\section{RESULTS}

A total of 25 out of 151 patients experienced RILD after 3D-CRT, including 17 patients with HCC and 8 patients with GC. In univariate testing, HBV carrier status ( 22 of 76 vs. 3 of $75, p<0.001$ ), mean liver radiation dose ( $21.4 \mathrm{~Gy}$ vs. $17.5 \mathrm{~Gy}$, $p=0.006$ ), and the volume fraction that received more than 30
Gy $(34.6 \%$ vs. $26.6 \%, p=0.01)$ were the statistically significant factors associated with RILD (Table 2). These 3 factors were selected for the subsequent multivariate test; the continuous variables were entered for the 2 dose-volume factors. In multivariate analysis, HBV carrier status was the only independent factor (hazard ratio $=4.02, p<0.001$ ). Among the 22 HBV carriers who had RILD, serum HBV DNA quantification test results were available for 6 patients during their RILD. All 6 patients showed serologic evidence of viral hepatitis B reactivation (serum HBV DNA higher than $5 \mathrm{pg} / \mathrm{mL}$ ) at the time of active RILD.

The best estimates of the 4 parameters in the parallelarchitecture NTCP model, $\mathrm{v}_{50}, \sigma, \mathrm{D}_{50}$, and $k$, were 0.54 , $0.14,50 \mathrm{~Gy}$, and 0.18 , respectively, for the whole group (151 patients). We divided patients into 2 subgroups by HBV carrier status, the statistically significant factor in multivariate testing. The parameters were $0.53,0.07,50 \mathrm{~Gy}$, and $4.6 \times 10^{-7}$, respectively, for patients who were HBV carriers. In contrast, the parameters were $0.59,0.12,25 \mathrm{~Gy}$, 
Table 2. Univariate analysis of patient-related, treatment-related, and dosimetric factors associated with radiation-induced liver disease (RILD)

\begin{tabular}{|c|c|c|c|}
\hline Variable & RILD & No RILD & $p$ \\
\hline Gender & & & 0.19 \\
\hline Male & 20 & 84 & \\
\hline Female & 5 & 42 & \\
\hline Malignancy & & & 0.31 \\
\hline Hepatocellular carcinoma & 17 & 72 & \\
\hline Gastric carcinoma & 8 & 54 & \\
\hline \multicolumn{4}{|l|}{ Hepatitis B carrier } \\
\hline Yes & 22 & 54 & $<0.001$ \\
\hline No & 3 & 72 & \\
\hline Cirrhosis of liver grading & & & 0.051 \\
\hline Child-Pugh class A & 18 & 112 & \\
\hline Child-Pugh class B & 7 & 14 & \\
\hline Transcatheter arterial chemoembolization & & & 0.87 \\
\hline Yes & 13 & 61 & \\
\hline No & 12 & 65 & \\
\hline Isocenter dose (Gy) & $48.5 \pm 4.0$ & $47.9 \pm 5.6$ & 0.61 \\
\hline Normal liver volume $(\mathrm{mL})$ & $1141.7 \pm 295.0$ & $1157.8 \pm 313.3$ & 0.81 \\
\hline Age & $56.5 \pm 9.5$ & $54.7 \pm 13.5$ & 0.44 \\
\hline Mean liver dose $(\mathrm{Gy})$ & $21.4 \pm 17.5$ & $17.5 \pm 6.3$ & 0.006 \\
\hline Volume fraction receiving more than $30 \mathrm{~Gy}(\%)$ & $34.6 \pm 15.8$ & $26.6 \pm 13.5$ & 0.01 \\
\hline
\end{tabular}

and 59.8, respectively, for patients who were not HBV carriers (Table 3).

The partial-volume response with the best-fit model parameters of the whole group, HBV carriers, and non-HBV carriers is shown in Fig. 1. A threshold effect with an irradiated partial volume of 0.4 to 0.6 seems to exist. The dose responses with the parameters for the whole group and the subgroups are shown in Fig. 2. The threshold effect was again seen in the non-HBV carrier subgroup but not in the HBV carrier subgroup. This distinction was derived from the difference in the slope parameter of dose-response curves between the 2 subgroups. When the observed complication rate is compared with fitted cumulative functional reserve, the correlation of complication probability with fraction of liver damaged expected from the model is as shown in Fig. 3. More applicable threshold effects appeared in both subgroups than in the whole group. No patient in either subgroup with a fraction damaged less than 0.4 experienced RILD.

In the HBV carrier subgroup, the goodness-of-fit deviance for the estimated HBV parameter set was 90.21 ( $p>$ $0.95)$. The deviance $(58.73, p<0.2)$ was much worse when we used the non-HBV parameter set for the HBV carrier subgroup. Similarly, in the non-HBV carrier subgroup, de- viance for the non-HBV parameter set was $102.11(p>$ $0.99)$. The deviance $(63.0, p<0.3)$ would have been worse if we had used the HBV parameter set in the non-HBV carrier subgroup.

\section{DISCUSSION}

With integration of 3D-CRT into a treatment modality for patients with hepatic and upper gastrointestinal malignancies, higher doses of radiation can be delivered to the liver (15). Dose escalation resulted in improved intrahepatic disease control in series from both South Korea and the University of Michigan $(16,17)$. RILD inevitably became the most important complication and deserved attention in a certain proportion of patients from each series. Our previous study indicated the significantly higher susceptibility of HBV carriers to RILD among a group of HCC patients undergoing 3D-CRT (6). Review of laboratory data from our most recent patients with RILD (treated after 2001) disclosed serologic evidence of HBV reactivation simultaneously with their active RILD. Such evidence implies biologic impact on the development of RILD in HBV carrier patients. A similar phenomenon of $\mathrm{HBV}$ reactivation has been observed in a series of patients who received

Table 3. The parameters of parallel-architecture NTCP model for all patients and the subgroups

\begin{tabular}{lcccc}
\hline $\begin{array}{c}\text { Best estimate of parameter } \\
\text { (95\% confidence interval) }\end{array}$ & \multicolumn{1}{c}{$\sigma$} & $\mathrm{D}_{50}$ & $k$ \\
\hline Whole group (151 patients) & $0.54(0.51-0.58)$ & $0.14(0.11-0.16)$ & $50 \mathrm{~Gy} \mathrm{(24-110)}$ & $\begin{array}{c}0.18(0.11-0.27) \\
\text { HBV carriers (76 patients) }\end{array}$ \\
Non-HBV carriers (75 patients) & $0.53(0.51-0.55)$ & $0.073(0.05-0.15)$ & $50 \mathrm{~Gy}(0->100)$ & $4.56 \times 10^{-7}(<0-0.06)$ \\
\hline
\end{tabular}

Abbreviation: $\mathrm{HBV}=$ hepatitis $\mathrm{B}$ virus. 


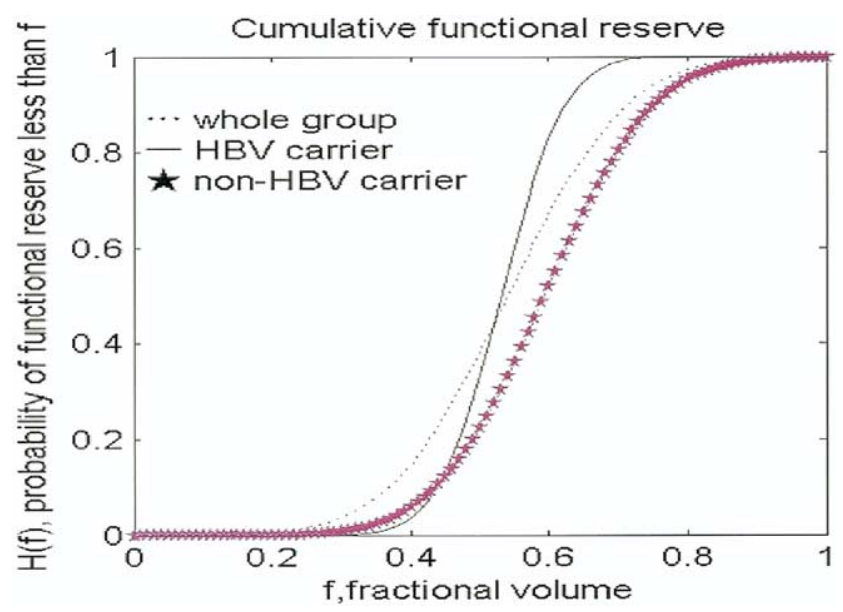

Fig. 1. The partial-volume response at high-dose limit with the best-fit model parameters in the whole group, hepatitis B virus (HBV) carrier group, and non-HBV carrier group.

chemotherapy (18). Whether radiation activates the process of HBV replication that presents as RILD in patients undergoing 3D-CRT warrants further investigation.

Several dosimetric parameters have been reported to be associated with development of RILD. Lawrence et al. (4) first showed the significance of the correlation between mean liver dose and RILD. They also tried parameterization of the Lyman NTCP model and found a difference in volume effect of partial-liver irradiation from the original report on whole-liver irradiation (19). Dawson et al. (5) updated model parameters with a larger group of recruited patients and confirmed the large volume effect in partialliver irradiation. We previously reported results for 17 of 89 HCC patients with RILD and performed Lyman NTCP model parameterization (6). For our patients who were not HBV carriers, similar to those reported in the Michigan series, the volume effect was as large as expected.

In this study, we included patients with diagnoses of HCC or GC. All patients underwent standard procedure for simulation and dose-volume calculation of the liver, regardless of treatment intent (i.e., definitive radiotherapy or postgastrectomy adjuvant radiotherapy). Despite the heterogeneity of treatment combined with radiation (e.g., chemotherapy) or treatment preceding 3D-CRT (e.g., chemoembolization) in these 2 diseases, clinical observations and univariate testing precluded their adjunct interference in the development of RILD. When patients were grouped by the nondosimetric biologic factor of HBV carrier status, a big difference in the slope parameter of the dose-response curve was found. The curve for non-HBV carriers was steep, and the curve for HBV carriers was shallow. Among the 2 subgroups, the fits were proved acceptable for each subgroup itself but were much worse with the estimates from the other subgroup. The results reveal that the estimates from the parallel-architecture NTCP model are more effective after patients are grouped by significant nondosimetric factors. In the rank-plan plots (Fig. 3), in order of risk for RILD, a threshold for fraction damaged occurred around 0.4 for the
2 subgroups. Hence, the threshold was not as clear in the plots of the whole group. This subgroup threshold was consistent with modeling work by Jackson et al. (7). This observation means that stratification of patients by HBV carrier status improved parameterization of the parallelarchitecture NTCP model. However, all but 1 HBV carrier patient had a fraction-damaged value of more than 0.4. In contrast, most of the non-HBV carrier patients had a fraction-damaged value of less than 0.4. The similar thresholds in both subgroups but the distribution difference in fraction damaged partly explains the effectiveness in grouping patients by this nondosimetric factor and emphasizes the importance in biologic impact on the pathogenesis of RILD.

NTCP parameterization is model dependent. The estimates were generated exactly from the modeling process of available patient data by maximum-likelihood method. The heterogeneities within patients may confound the estimation and widen the confidence interval. Clinical or biologic factors are the most likely sources of these interferences. Dawson et al. (5) demonstrated the significance of disease origin of intrahepatic malignancies, chemotherapy regimen, and gender in susceptibility to RILD. The estimates differed between patients with hepatobiliary malignancy and those with liver metastasis. Yorke et al. (8) found the correlation of dosimetric and NTCP models with radiation pneumonitis was stronger for the lower portions of the lungs and the ipsilateral lung. Our previous series with HCC patients disclosed HBV carrier and cirrhosis of the liver as significant factors with impact on RILD (6). The volume-effect parameter of the Lyman NTCP model was much smaller in HBV carriers, indicating poor tolerance to partial-liver irradiation. In this study, the slope parameter of the doseresponse curve was as small as $4.56 \times 10^{-7}$ for $\mathrm{HBV}$ carrier patients. Such a shallow dose-response curve implies the certain risk of RILD with even a very low dose of radiation. With the unique parameters derived from the HBV carrier subgroup, the calculated fraction damaged was close to the

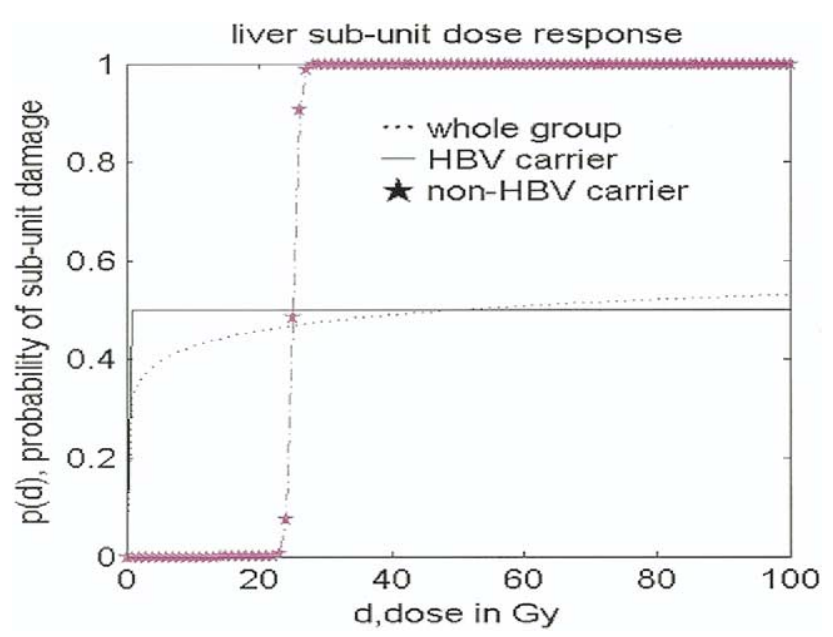

Fig. 2. The dose-response at whole volume irradiated with the best-fit model parameters in the whole group, hepatitis B virus (HBV) carrier group, and non-HBV carrier group. 


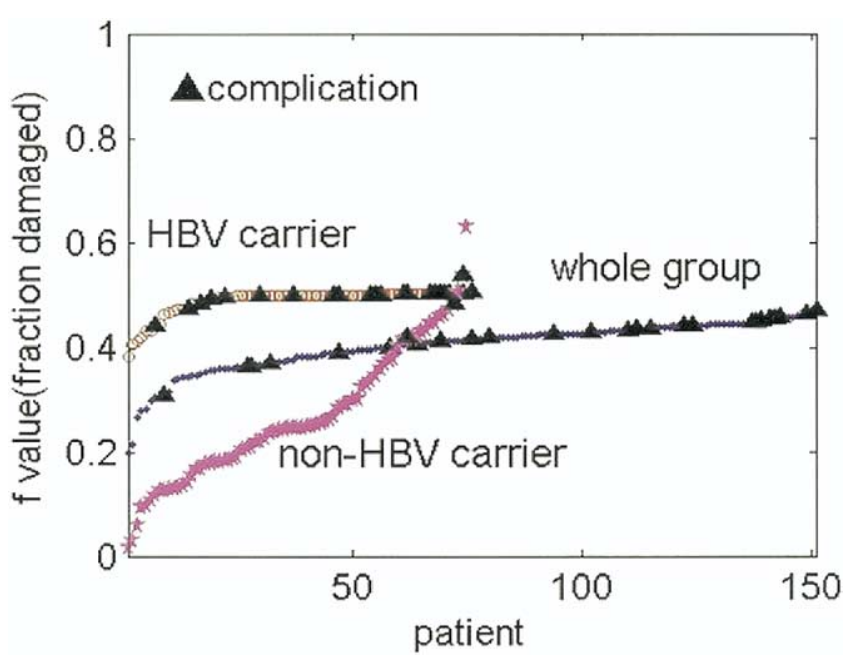

Fig. 3. Patient ranked based on calculated fraction damaged with the best-fit model parameters in the whole group, hepatitis B virus (HBV) carrier group, and non-HBV carrier group.

threshold for development of RILD under a low dose of radiation. This finding raises the possibility of a biologic pathway triggered by radiation that contributes to development of RILD. The NTCP model itself is still a powerful tool by which to estimate the risk of RILD if significant nondosimetric factors have been used to reduce heterogeneity among patient subgroups.

The parallel-architecture NTCP model was initiated by Jackson and colleagues (12). The basic assumption of this model is that the functional subunits of a parallel organ contribute to function independently of each other. The probability concept of functional-subunit damage was proposed by Niemierko and Goitein (20). Radiation-induced lung injury has been most frequently tested by application of a parallel model $(8,21)$. The appropriate fits for observed organ damage with the calculated parallel model may indicate functional subunits of parallel configuration. Jackson and colleagues (12) examined this model with clinical data from patients with intrahepatic malignancies who were undergoing 3D-CRT (7). The fits and estimates of their data support the existence of parallel features in the liver. Parallel organ architecture has also been confirmed with the same database that shows a large volume effect with the
Lyman NTCP model $(4,5)$. Small fractions of such organs can be safely irradiated with high doses and result in no complication at all $(22,23)$. Whole-liver irradiation was not used in our study. The estimates of non-HBV carrier patients with partial-liver irradiation came close to those in reported series. The fits of our data with the parallel-architecture NTCP model better described the parallel features of liver in the 2 subgroups on the basis of HBV carrier status. The difference between the 2 subgroups in tolerance of functional subunits to radiation may arise from the underlying biologic susceptibility of HBV carriers.

This study had a few limitations. Most patients were treated in the era of systems that did not allow respiratory gating. Some subunits of liver moved between high-dose and low-dose regions during radiotherapy. The static simulation images and reconstructions could not take these movements into account and possibly caused some errors in the modeling work. In addition, the number of patients in each subgroup was relatively small. Thus, estimates and confidence intervals may not be concise because of few events and patients. The fraction size and treatment protocol were not the same as those used in reported series. A direct comparison of estimated parameters may not be feasible in this situation. With partial-liver irradiation, the current NTCP model assumes homogeneous function in each part of the liver. We did not use any functional examination with spatial resolution in this study, and we accepted this homogeneous assumption in our parameterization. The conformal radiation design and uneven functional distributions within the liver, if they exist, may confound the estimation of the NTCP model.

In conclusion, the parallel-architecture NTCP model remains effective in predicting the risk of RILD after stratifying patients by the significant nondosimetric factor of HBV carrier status. The threshold fraction of volume damaged was around 0.4 in both subgroups, with their corresponding estimates. The main difference in model parameters between HBV carriers and non-HBV carriers was the slope parameter of the dose-response curve, which implies an underlying biologic impact on RILD in HBV carriers. The nondosimetric impact on radiation-induced complication needs to be clarified before accurate NTCP parameterization can be done.

\section{REFERENCES}

1. Lawrence TS, Robertson JM, Anscher MS, et al. Hepatic toxicity resulting from cancer treatment. Int J Radiat Oncol Biol Phys 1995;31:1237-1248.

2. Cheng JC, Wu J-K, Huang C-M, et al. Radiation-induced liver disease after radiotherapy for hepatocellular carcinoma: Clinical manifestation and dosimetric description. Radiother Oncol 2002;63:41-45.

3. Cheng JC, Wu J-K, Huang C-M, et al. Radiation-induced liver disease after three-dimensional conformal radiotherapy for patients with hepatocellular carcinoma: Dosimetric analysis and implication. Int J Radiat Oncol Biol Phys 2002;54:156162.

4. Lawrence TS, Ten Haken RK, Kessler ML, et al. The use of
3-D dose volume analysis to predict radiation hepatitis. Int $J$ Radiat Oncol Biol Phys 1992;23:781-788.

5. Dawson LA, Normolle D, Balter JM, et al. Analysis of radiation-induced liver disease using the Lyman NTCP model. Int J Radiat Oncol Biol Phys 2002;53:810-821.

6. Cheng JC, $\mathrm{Wu} \mathrm{J-K}$, Lee PC, et al. Biological susceptibility of hepatocellular carcinoma patients treated with radiotherapy to radiation-induced liver disease. Int J Radiat Oncol Biol Phys 2004;60: 1502-1509.

7. Jackson A, Ten Haken RK, Robertson JM, et al. Analysis of clinical complication data for radiation hepatitis using a parallel architecture model. Int J Radiat Oncol Biol Phys 1995; 31:883-891. 
8. Yorke ED, Jackson A, Rosenzweig KE, et al. Dose-volume factors contributing to the incidence of radiation pneumonitis in non-small-cell lung cancer patients treated with three-dimensional conformal radiation therapy. Int J Radiat Oncol Biol Phys 2002;54:329-339.

9. Cheng JC, Chuang VP, Cheng SH, et al. Unresectable hepatocellular carcinoma treated with radiotherapy and/or chemoembolization. Int J Cancer 2001;96:243-252.

10. Smalley SR, Gunderson L, Tepper J, et al. Gastric surgical adjuvant radiotherapy consensus report: Rationale and treatment implementation. Int J Radiat Oncol Biol Phys 2002;52: 283-293.

11. Trotti A, Byhardt R, Stetz J, et al. Common Toxicity Criteria: Version 2.0. An improved reference for grading the acute effects of cancer treatment: Impact on radiotherapy. Int $J$ Radiat Oncol Biol Phys 2000;47:13-47.

12. Jackson A, Kutcher GJ, Yorke ED. Probability of radiation induced complications for normal tissues with parallel architecture subject to nonuniform irradiation. Med Phys 1993;20: 613-625.

13. Cox D, Snell E. Analysis of binary data. New York: Chapman and Hall, 1989. p. 179-184.

14. Roberts SA, Hendry JH. The delay before onset of accelerated tumour cell repopulation during radiotherapy: A direct maximum-likelihood analysis of a collection of worldwide tumourcontrol data. Radiother Oncol 1993;29:69-74.

15. Ten Haken RK, Lawrence TS, McShan DL, et al. Technical considerations in the use of 3-D beam arrangements in the abdomen. Radiother Oncol 1991;22:19-28.
16. Park H, Seong J, Han K, et al. Dose-response relationship in local radiotherapy for hepatocellular carcinoma. Int J Radiat Oncol Biol Phys 2002;54:150-155.

17. Dawson LA, McGinn CJ, Normolle D, et al. Escalated focal liver radiation and concurrent hepatic artery fluorodeoxyuridine for unresectable intrahepatic malignancies. J Clin Oncol 2000;18:2210-2218.

18. Yeo W, Chan PKS, Chan HLY, et al. Hepatitis B virus reactivation during cytotoxic chemotherapy-enhanced viral replication precedes overt hepatitis. J Med Virol 2001;65:473477.

19. Burman C, Kutcher GJ, Emami B, et al. Fitting of normal tissue tolerance data to an analytic function. Int $J$ Radiat Oncol Biol Phys 1991;21:123-135.

20. Niemierko A, Goitein M. Modeling of normal tissue response to radiation: The critical volume model. Int J Radiat Oncol Biol Phys 1993;25:135-145.

21. Kwa SL, Theuws JC, Wagenaar A, et al. Evaluation of two dose-volume histogram reduction models for the prediction of radiation pneumonitis. Radiother Oncol 1998;48:61-69.

22. Yorke ED, Kutcher GJ, Jackson A, et al. Probability of radiation-induced complications in normal tissues with parallel architecture under conditions of uniform whole or partial-organ irradiation. Radiother Oncol 1993;26:226237.

23. Zaider M, Anols HI. A little to a lot or a lot to a little: Is NTCP always minimized in multiport therapy? Int J Radiat Oncol Biol Phys 1998;41:945-950. 